the effectiveness of physical activity. The presence in patients with obesity and overweight polymorphisms of genes that adversely affect carbohydrate and fat metabolism, dictates the need to organize a special personalized approach to diet therapy and exercise.

\section{P291 NEPHROGENIC DIABETES INSIPIDUS IN A FEMALE INFANT}

Kerry Noy*, Mohamad Hilal, Jayabharathi Sakamudi.

\subsection{6/archdischild-2019-epa.641}

\section{Prince Charles Hospital, Merthyr Tydfil, UK}

Aim Nephrogenic Diabetes Insipidus(NDI) is rare. $90 \%$ of cases are due to a defect in the AVPR2 gene which is widely believed to be inherited in an X-linked recessive pattern; from asymptomatic carrier mothers, to severely affected sons, but not daughters. Other, less common cases ( $\sim 1 \%$ of NDI cases), are inherited in an autosomal dominant pattern due to a defect in the AQP2 gene. However, our case will demonstrate how females can present with symptomatic NDI secondary to AVPR2 mutation.

Methods This is a case study of a 14-month-old girl who was referred to the Paediatric Outpatient Department with a history of faltering growth, excessive drinking and plentiful wet nappies. At birth she plotted on the 50th centile for weight but subsequently dropped to the 9th. Weaning was a difficult, with her preferring liquids to solids. Moreover, excessive thirst resulted in her drinking water from the swimming pool, bath water and even the dog's bowl. Serum electrolytes, in addition to serum and urine osmolality and a desmopressin test, confirmed the diagnosis of NDI.

There was positive family history with the patient's Father having NDI, treated initially with diuretics then desmopressin until age 14, and since no longer requiring medication. An assumption was made that the patient thus acquired NDI via autosomal dominant inheritance of AQP2 gene. However, genetic tests were not performed at this stage.

Results The patient's Father was found to be hemizygous for AVPR2 gene. Genetic testing then revealed the patient to be a carrier of AVPR2 gene. Inherited via X-linked recessive form this would not normally affect females. Nevertheless, due to lyonisation of $\times$ chromosome this patient was symptomatic.

Conclusion X-linked autosomal recessive disorders can also affect females. This is due to the process of lyonisation, which in itself has different forms and causes. Appreciation of lyonisation defect is crucial in the diagnosis and family counselling of patients with X-linked recessive disorders such as NDI, in addition to other more common disorders such as Glucose-6Phosphate Dehydrogenase Deficiency (G6PD), Duchenne Muscular Dystrophyor Haemophilia.

\section{P292 NEONATAL DIABETES IN A TERTIARY CENTRE; GENETICS DICTATING MANAGEMENT}

\footnotetext{
${ }^{1}$ Laura McCarron*, ${ }^{1}$ Eleanor Burke, ${ }^{1}$ Gillian O'Donnell, ${ }^{1}$ Eirin Carolan, ${ }^{1,2}$ Nuala Murphy,

${ }^{1,3}$ Ciara McDonnell. 'Department of Paediatric Endocrinology, Children's Hospital, Temple Street, Dublin , Ireland; ' ${ }^{2}$ chool of Medicine, University College Dublin, Dublin , Ireland; ${ }^{3}$ Discipline of Paediatrics, University of Dublin, Trinity College, Dublin , Ireland
}

10.1136/archdischild-2019-epa.642
Background Neonatal diabetes mellitus is a rare condition with one case per 300,000 to 500,000 live births. It presents with marked hyperglycaemia in the first six months of life and is commonly of generic origin. Approximately half of cases are transient (TNDM) with the reminder being permanent (PNDM). The majority of Permanent Diabetes Mellitus (PNDM) cases are secondary to genetic mutations in K-ATP channel genes.

Case We report two infants who presented in the neonatal period with hyperglycaemia and were subsequently diagnosed with neonatal diabetes.

The first infant presented at 22 hours of life with blood glucose of $44.3 \mathrm{mmol} / \mathrm{L}$ and in ketoacidosis. He was born by LSCS at 34 weeks gestation due to IUGR with a birth weight of $1.36 \mathrm{~kg}$. He received an IV insulin infusion for two months with frequent dose titration due to challenging glycaemic control. He was then successfully transitioned to a subcutaneous insulin infusion pump. Genetic analysis confirmed homozygous INS non sense variant, p(ARf43Ter).

The second infant presented at six weeks of age in severe diabetic ketoacidosis with a blood glucose of $60 \mathrm{mmol} / \mathrm{L}$. She was born at term weighing $2.46 \mathrm{~kg}$ following induction of labour for IUGR. She presented with lethargy and vomiting for 24 hours on a background of failure to thrive and chronic candidiasis from birth. She was managed with an IV insulin infusion in the ICU before transitioning to a subcutaneous insulin pump. Genetic analysis revealed a de novo KCNJ11 gene mutation. She was then successfully transitioned to oral Glibenclamide which optimised her glycaemic control and resulted in complete withdrawal of subcutaneous insulin.

Discussion These are two cases of NDM with identified genetic mutations. The INS mutation is localised to amino acid residues affecting cleavage and/or folding of pre-proinsulin and pro-insulin which leads to prolonged ER stress and subsequent B-cell apoptosis. The KCJN11 gene mutation identified in our second patient results in K-ATP channel overactivity. The K-ATP channel is at the end of the glycolytic pathway and has a KIR amino terminus responsible for channel gating. Its deletion results in channels that are continuously closed reducing sensitivity to inhibitory ATP and resulting in dramatic hyperglycaemia. Oral glibenclamide inhibits K-ATP channel activity with resultant normoglycaemia. Early identification is essential for optimal management and targeted therapy.

\section{P293 AN AUDIT OF PREGNANCY OUTCOMES IN WOMEN WITH CHILDHOOD ONSET TYPE 1 DIABETES MELLITUS}

${ }^{1}$ Roy Gavin Stone* ${ }^{2}$ Emma Troy, ${ }^{3}$ Paul Scully, ${ }^{1}$ Anne Quinn, ${ }^{4}$ Yvonne Moloney, ${ }^{1}$ Orla Neylon, ${ }^{4}$ John Slevin, ${ }^{2}$ Eoin Noctor, ${ }^{1}$ Anne Marie Murphy, ${ }^{1}$ Clodagh O'Gorman. ${ }^{1}$ Paedaitrics Department, University Hospital Limerick, Limerick, Ireland; ${ }^{2}$ Department of General Medicine, University Hospital Limerick, Limerick, Ireland; ${ }^{3}$ Paediatric Department, University Hospital Limerick, Limerick, Ireland; ${ }^{4}$ Department of Obstetrics and Gynaecology, University Maternity Hospital Limerick, Limerick, Ireland

\subsection{6/archdischild-2019-epa.643}

Background Pregnancy in women with type 1 diabetes mellitus is associated with an increased risk of congenital malformations, obstetric complications, and neonatal morbidity. In order to minimise morbidity for both mothers and infants there needs to be good interdisciplinary care between diabetologists, obstetricians, neonatologists and relevant nursing specialists. 
Aim To investigate maternal, perinatal, and neonatal outcomes of pregnancies in women with type 1 diabetes in the University Maternity Hospital Limerick, Ireland.

Methods This retrospective descriptive study was conducted from charts identified from the diabetes in pregnancy register from $1^{\text {st }}$ July 2007 to $1^{\text {st }}$ July 2017 at University Maternity Hospital Limerick.

Results 17 women were identified from the register with juvenile onset ( $<18$ years) type 1 diabetes mellitus during the 10 year study period who gave birth to 23 live infants. Maternal diabetes related medical conditions included; diabetic retinopathy (29\%), hypertension (11.8\%), recurrent urinary tract infections $(11.8 \%)$, diabetic nephropathy (11.8\%), poor hypoglycaemia awareness (11.8\%), diabetic neuropathy $(5.9 \%)$ and cataracts $(5.9 \%) .11 .7 \%$ women had a past history of illicit drug use. $8.7 \%$ women were smoking at the time of booking. Antenatal obstetric complications included polyhydramnios $(17.4 \%)$, intrauterine growth restriction $(17.4 \%)$, pre-eclampsia (8.7\%), maternal hypoglycaemic seizure $(4.3 \%)$ and pregnancy induced hypertension (4.3\%). 39.1\% infants were born to primiparous women. $87 \%$ infants were bottle-fed. $73.9 \%$ births were unplanned pregnancies. Of $n=5$ infants born via vaginal delivery there were no cases of shoulder dystocia. $60.9 \%$ neonates were male. The mean gestation was 37.2 weeks (range 33.3 to 39.4 ). The mean birth weight was $3.57 \mathrm{~kg}$ (range 2.01 to 5.54). The median APGAR at 1 minute was 9 (range 5-9). The median APGAR at 5 minutes was 10 (range 4-10). $17.4 \%$ neonates required free flow $\mathrm{O} 2$ at birth and $8.7 \%$ required bag-mask ventilation. $95.7 \%$ neonates were admitted to the neonatal unit. Of the $n=23$ infants studied medical issues included; hypoglycaemia (60.9\%), jaundice requiring phototherapy $(43.5 \%)$, transient tachypnoea of the newborn (39.1\%), prematurity (30.4\%), macrosomia (17.4\%), low birth weight $(13 \%)$, poor feeding $(13 \%)$, polycythaemia $(8.7 \%)$, trisomy $21(4.3 \%)$, respiratory distress syndrome $(4.3 \%)$ and hyponatremia $(4.3 \%)$.

Conclusion Neonates born to mothers with juvenile onset T1DM should be considered a high risk group and clinicians should have a low threshold for admission to the neonatal unit.

\section{P294 CUSHING SYNDROME AND ADRENAL INSUFFICIENCY INDUCED BY HIGH DOSE PROLONGED INTRANASAL BETAMETHASONE}

${ }^{1}$ Claire Reynolds*, 'Pankaj Agrawal, ${ }^{1}$ Aisling McCann, ${ }^{2}$ Triona O'Sullivan, ${ }^{3}$ Muireann Ni Chroinin, 'Stephen MP O'Riordan. 'Department of Paediatric and Endocrinology, Cork University Hospital, Cork, Ireland; ${ }^{2}$ Pharmacy Department, Cork University Hospital, Cork, Ireland; ${ }^{3}$ Department of Paediatric and Respiratory Medicine, Cork University Hospital, Cork, Ireland

\subsection{6/archdischild-2019-epa.644}

Iatrogenic Cushing syndrome and secondary adrenal insufficiency due to intranasal steroids is rare in children. A 7 year old boy was referred to the paediatric endocrine clinic with suspected Cushing syndrome. He presented with excessive weight gain, marked striae and hypertension. His GP had performed a morning cortisol $<28 \mathrm{nmol} / \mathrm{L}(171-800 \mathrm{nmol} / \mathrm{L})$. He had a background history of obstructive sleep apnoea for which he had been prescribed Betnesol 0.1\% (Betamethasone sodium phosphate $1 \mathrm{mg} / \mathrm{ml}$ ) nasal drops and Montelukast 4 mg daily. Physical examination revealed hypertrichosis on his back, buffalo-humped neck, and purple striae on his legs and inner thighs. He was Tanner stage 1, normal genitalia. Auxology: weight $46.1 \mathrm{~kg}\left(>99.6^{\text {th }}\right.$ centile); height $124.6 \mathrm{~cm}\left(50^{\text {th }}\right.$ $75^{\text {th }}$ centile), body mass index (BMI) $30 \mathrm{~kg} / \mathrm{m} 2\left(>99.6^{\text {th }}\right.$ centile) and blood pressure was $124 / 72 \mathrm{mmHg}$ ( $>99^{\text {th }}$ centile). The drug history was reviewed in detail by our Paediatric Pharmacist and revealed that over the past 20 months, he had been using betamethasone nasal drops continuously, prescribed at a dose of 2 drops, twice daily, into each nostril. A total of 38 repeat prescriptions had been dispensed and he had been taking double the prescribed amount per month. The duration of use (20 months) also far exceeded the recommended indication (4-6weeks max). Laboratory investigations: blood glucose $4.7 \mathrm{mmol} / \mathrm{L}$, early morning cortisol $<28 \mathrm{mmol} / \mathrm{L}(171-$ $800 \mathrm{nmol} / \mathrm{L})$ and $\mathrm{ACTH}<0.2$ undetectable $(1.1-13.2 \mathrm{pmol} / \mathrm{L})$. Renin and aldosterone, anti-21 hydroxylase antibodies, thyroid function tests, IGF-1, coeliac screen, and renal/liver/bone were all normal. A short synacthen test (250micrograms) confirmed HPA axis suppression for our patient (cortisol concentrations: $<28 \mathrm{nmol} / \mathrm{L}$ at time: 0 mins, $30 \mathrm{mins}$ and $60 \mathrm{mins}$ ). This is gold standard diagnostic test when adrenal suppression is suspected. He was immediately commenced on replacement hydrocortisone therapy at a dose of $8-10 \mathrm{mg} / \mathrm{m}^{2} /$ day. He was also educated on emergency hydrocortisone administration and stress dosing. He will continue on hydrocortisone until his HPA axis recovers. Betamethsone is a potent steroid 25-30 times more potent than hydrocortisone

Conclusion We describe a case of iatrogenic Cushing syndrome and adrenal insufficiency secondary to administration of longterm high-dose betamethasone nasal drops. Very few cases to date have been reported in the literature in children. Cushing syndrome caused by intranasal steroids is a rare event and more commonly occurs after oral or topical steroid use. Our patient was taking double the recommended dose for more than 20 months. Paediatricians must be aware of the complications of nasal steroid administration and long-term use should be avoided

\section{P295 CHARACTERISTICS OF A POPULATION OF 311 PATIENTS OF BOTH SEXES WITH EARLY ONSET PUBERTAL SIGNS. DESCRIPTIVE STUDY}

${ }^{1}$ Paula Sol Ventura Wichner, 'Xavier Herrero, 'Maria Laura Llorca, 'Agustina Magistris, 'Zelmira Bosch, ${ }^{1}$ Marisa Torres Lacruz, ${ }^{2}$ Georges Denis Akel Perez ${ }^{*}$. ${ }^{1}$ Hospital de Nens, Barcelona, Spain; ${ }^{2}$ Paediatric Unit of Fundación Hospital de Nens de Barcelona, Barcelona, Spain

\subsection{6/archdischild-2019-epa.645}

Objective To assess anthropometric, biochemical and imaging studies (bone age, pelvic ultrasound and MRI) in patients who consulted the Endocrinology Department of the Hospital de Nens de Barcelona, referred by pediatricians to discard precocious puberty.

Methodology Retrospective descriptive study based on review of medical records, with first consultation between 2010 and 2018. Search from keyword in the diagnosis: puberty. The subjects were separated in 6 groups: involuted precocious breast development, early non-progressive breast development, central precocious puberty (PPC), peripheral precocious 